\title{
VIBRATION ANALYSIS OF PRE-TWISTED BEAMS USING THE SPLINE COLLOCATION METHOD
}

Ming-Hung Hsu

Department of Electrical Engineering, National Penghu University, Penghu, Taiwan., hsu@npu.edu.tw

Follow this and additional works at: https://jmstt.ntou.edu.tw/journal

Part of the Electrical and Computer Engineering Commons

\section{Recommended Citation}

Hsu, Ming-Hung (2009) "VIBRATION ANALYSIS OF PRE-TWISTED BEAMS USING THE SPLINE COLLOCATION METHOD," Journal of Marine Science and Technology. Vol. 17: Iss. 2, Article 4.

DOI: 10.51400/2709-6998.1964

Available at: https://jmstt.ntou.edu.tw/journal/vol17/iss2/4

This Research Article is brought to you for free and open access by Journal of Marine Science and Technology. It has been accepted for inclusion in Journal of Marine Science and Technology by an authorized editor of Journal of Marine Science and Technology. 


\title{
VIBRATION ANALYSIS OF PRE-TWISTED BEAMS USING THE SPLINE COLLOCATION METHOD
}

\author{
Ming-Hung Hsu*
}

Key words: natural frequency, pre-twisted beam, spline collocation method, tapered beam, boundary condition.

\begin{abstract}
The variation in the accuracy of the calculated natural frequencies of pre-twisted beams solved with spline collocation method is investigated in this study. The spline collocation method is used to formulate the eigenvalue problems of pre-twisted beams. Three types of boundary conditions are considered. Numerical results indicate that the accuracy of the calculated natural frequencies is significantly dependent upon the pre-twisted angle of the non-uniform beam. Results that show that spline collocation method is very competitive for the vibration analysis of pre-twisted beams are presented.
\end{abstract}

\section{INTRODUCTION}

Speaking of many kinds of design, dynamic characteristics of pre-twisted beams absolutely play a vital role. In the field of turbo or compressor engineering, for simplicity, the beam is frequently approximated as a pre-twisted beam. At the design stage, accurate prediction of national frequencies of nonuniform pre-twisted beam is of considerable importance at the design stage. The coupled natural frequencies of a pre-twisted beam were also investigated using a number of different methods. Abrate [1] studied the vibration of a pre-twisted blade using the Rayleigh-Ritz method. Anderson [2] studied the flexural vibration of rotating bars. Dawson $[4,5]$ studied the vibration of a pre-twisted blade using the Rayleigh-Ritz method. Gupa and Rao [8] applied the finite element method for finding the variation of natural frequencies of doubly tapered and twisted Timoshenko beams. Hodges et al. [9] used the transfer matrices to compute the fundamental frequencies and corresponding modal displacements along the non-uniform rotating beams. They displayed that a blade has a complex geometry that makes an exact investigation of its characteristics somewhat complex. Kuang and Hsu $[10,11]$ presented that the blade is frequently approximated as a pre-twisted

Paper submitted 11/08/07; accepted 04/07/08. Author for correspondence: Ming-Hung Hsu (e-mail: hsu@npu.edu.tw).

*Department of Electrical Engineering, National Penghu University, Penghu, Taiwan. beam for simplicity in the field of turbo or compressor engineering. Lin et al. [12] presented the accurate modified transfer matrix method for studying the dynamic behavior of a non-uniform pre-twisted Timoshenko beam. Rao $[15,16]$ studied the natural frequency of pre-twisted beam to consider the complex shape of beam. They presented the vibration problems of wind blades and turbo blades are crucial parts of the design. Storti and Aboelnaga [19] studied the transverse deflections of a straight tapered symmetric beam attached to a rotating hub as a model for the bending vibration of blades in turbomachinery. Subrahmanyam et al. [20] showed coupled bending-bending vibrations of pre-twisted cantilever blading allowing for shear deflection and rotary inertia by the Reissner method. Subrahmanyam and Rao [21] presented coupled bending-bending vibrations of pre-twisted tapered cantilever beams treated by the Reissner method. Swaminathan and Rao [22] solved the vibrations of rotating, pre-twisted and tapered blades. Young [27] dealt with the dynamics of a pre-twisted beam using Rao's comparison functions. In this work, the spline collocation method is implemented to formulate the eigenvalue problem of a pre-twisted beam in the discrete form. The integrity and computational efficiency of spline collocation method in this problem will be demonstrated through a series of case studies.

\section{SPLINE COLLOCATION METHOD}

The solutions to numerous complex pre-twisted beam problems have been efficiently obtained as the use of fast computers and range of available numerical methods, including the Galerkin method, differential quadrature method, finite element technique, differential transform, boundary element method, and Rayleigh-Ritz method. In this study, spline collocation method is employed to formulate the discrete eigenvalue problems of various pre-twisted beams. Prenter et al. [7, 14, 18] investigated spline and variation methods. Bert and Sheu [3] presented static analysis of beams and plates using spline collocation method. El-Hawary et al. [6] discussed quartic spline collocation methods for solving linear elliptic partial differential equations. They derived optimal quartic spline approximations to generated high order perturbations of partial differential equations. Patlashenko and Weller [13] applied the spline collocation method to solve two-dimensional problems, and determined the postbucking 
behavior of laminated panels subjected to mechanical and heating induced loadings. Viswanathan and Navaneethakrishnan [23] studied the free vibration of circular cylindrical thin shells using point collocation method. Rao and Kumar [17] presented a B-spline collocation method of higher order for a class of self-adjoint singularly pertured boundary value problems. Wu et al. [24, 25, 26] displayed the application of the spline collocation method to analysis of rigid frame structures under various loading conditions. Their results from the spline collocation method are compared with those obtained from the differential quadrature method, the finite element method, and other available analytical methods. The spline function can be derived from backward or central finite difference. In this work, we consider the knots $z_{i}$ as follows.

$$
z_{i}=\bar{a}+i h \text { for } i=-2,-1,0, \ldots, N+1, N+2
$$

where $z_{0}, z_{1}, z_{2}, \ldots, z_{N-1}, z_{N}$ are the abscissas of knots and $z_{-2}$, $z_{-1}, z_{N+1}, z_{N+2}$ are the abscissas of extended fictitious knots.

$$
h=\frac{\bar{b}-\bar{a}}{N}
$$

where distance $h$ between two adjacent knots keep constant. Spline function is given as follows $[3,6,13]$.

$$
\begin{aligned}
& B_{u i}(z)=B_{v i}(z)= \\
& \left\{\begin{array}{l}
\frac{\left(z-z_{i-3}\right)^{5}}{h^{5}} \text { if } z \in\left[z_{i-3}, z_{i-2}\right] \\
\frac{\left(z-z_{i-3}\right)^{5}-6\left(z-z_{i-2}\right)^{5}}{h^{5}} \text { if } z \in\left[z_{i-2}, z_{i-1}\right] \\
\frac{\left(z-z_{i-3}\right)^{5}-6\left(z-z_{i-2}\right)^{5}+15\left(z-z_{i-1}\right)^{5}}{h^{5}} \text { if } z \in\left[z_{i-1}, z_{i}\right] \\
\frac{\left(z-z_{i-3}\right)^{5}-6\left(z-z_{i-2}\right)^{5}+15\left(z-z_{i-1}\right)^{5}-20\left(z-z_{i}\right)^{5}}{h^{5}} \text { if } z \in\left[z_{i}, z_{i+1}\right] \\
\frac{\left(z-z_{i-3}\right)^{5}-6\left(z-z_{i-2}\right)^{5}+15\left(z-z_{i-1}\right)^{5}-20\left(z-z_{i}\right)^{5}+15\left(z-z_{i+1}\right)^{5}}{h^{5}} \quad \text { if } z \in\left[z_{i+1}, z_{i+2}\right] \\
\frac{\left(z-z_{i-3}\right)^{5}-6\left(z-z_{i-2}\right)^{5}+15\left(z-z_{i-1}\right)^{5}-20\left(z-z_{i}\right)^{5}+15\left(z-z_{i+1}\right)^{5}-6\left(z-z_{i+2}\right)^{5}}{h^{5}} \text { if } z \in\left[z_{i+2}, z_{i+3}\right] \\
0 \quad \text { otherwise }
\end{array}\right.
\end{aligned}
$$

where $B_{u,-2}(z), B_{u,-1}(z), B_{u, 0}(z), \ldots, B_{u, N+1}(z), B_{u, N+2}(z)$, and $B_{v,-2}(z), B_{v,-1}(z), B_{v, 0}(z), \ldots, B_{v, N+1}(z), B_{v, N+2}(z)$ form a basis for the function defined over the region $\bar{a} \leq z \leq \bar{b}$. The values at the knots are given by the following equations.

$$
\begin{aligned}
& U(z)=\sum_{i=-2}^{N+2} a_{u i} B_{u i}(z) \\
& V(z)=\sum_{i=-2}^{N+2} a_{v i} B_{v i}(z)
\end{aligned}
$$

where $a_{u i}$ and $a_{v i}$ are coefficients to be determined. There are $N+5$ collocation points in the domain. The spline functions should be at least one order higher than that of the governing differential equation so that accuracy and smoothness of the approximate solution can be guaranteed [24, 25, 26].

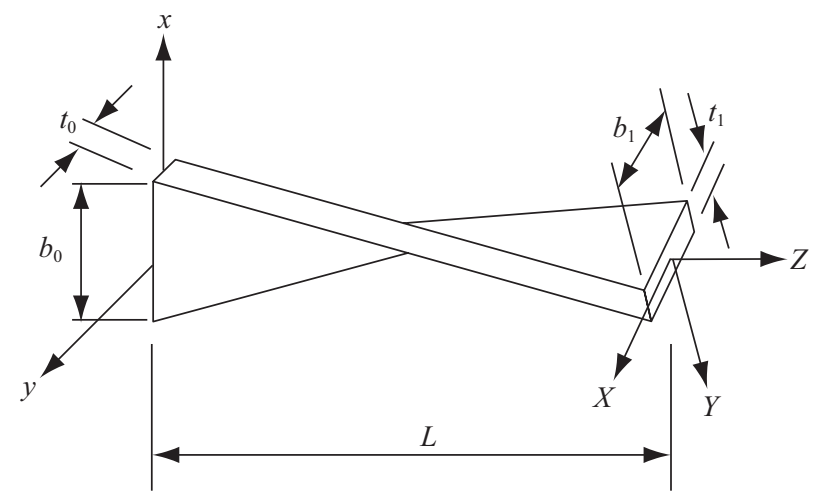

Fig. 1. Geometry of a pre-twisted beam.

\section{FORMATION OF THE EIGENVALUE PROBLEM}

The spline collocation method is employed to formulate the eigenvalue problems for pre-twisted beams. The pre-twisted beam is shown in Fig. 1.

The length of the pre-twisted beam is $L . b_{0}$ and $t_{0}$ denote the width and thickness of the pre-twisted beam at $z=0$, respectively. $\quad b_{1}$ and $t_{1}$ denote the width and thickness of the pretwisted beam at $z=L$, respectively. The deflection components $u$ and $v$ are the transverse flexible deflections of the pre-twisted beam. The kinetic energy of the beam, due to the lateral bending vibration $[2,10,11]$, is

$$
T=\frac{1}{2} \int_{0}^{L} \rho A\left(\left(\frac{\partial u}{\partial t}\right)^{2}+\left(\frac{\partial v}{\partial t}\right)^{2}\right) d z
$$

Consider the cross sectional area of the pre-twisted beam material at position $z$ to be

$$
A(z)=b_{0} t_{0}\left(1+\alpha \frac{z}{L}\right)\left(1+\beta \frac{z}{L}\right)
$$

where $\rho$ is the density of the pre-twisted beam, and the tapered angles of the pre-twisted beam are

$$
\begin{gathered}
\alpha=\frac{b_{1}-b_{0}}{b_{0}} \\
\beta=\frac{t_{1}-t_{0}}{t_{0}}
\end{gathered}
$$

The strain energy of the pre-twisted beam is $[2,10,11]$

$U=\frac{1}{2} \int_{0}^{L} E\left(I_{y y}\left(\frac{\partial^{2} u}{\partial z^{2}}\right)^{2}+2 I_{x y}\left(\frac{\partial^{2} u}{\partial z^{2}}\right)\left(\frac{\partial^{2} v}{\partial z^{2}}\right)+I_{x x}\left(\frac{\partial^{2} v}{\partial z^{2}}\right)^{2}\right) d z$

In this equation, $I_{x x}, I_{y y}$ and $I_{x y}$ are the area moments of inertia. Consider the tapered beam to be pre-twisted with a 
uniform pre-twisted angle $\theta_{t}$ and the moments of cross sectional area at the position $z$ can be derived as

$$
\begin{aligned}
& I_{x x}(z)=I_{X X} \cos ^{2}\left(\frac{z}{L} \theta_{t}\right)+I_{Y Y} \sin ^{2}\left(\frac{z}{L} \theta_{t}\right) \\
& I_{y y}(z)=I_{X X} \sin ^{2}\left(\frac{z}{L} \theta_{t}\right)+I_{Y Y} \cos ^{2}\left(\frac{z}{L} \theta_{t}\right) \\
& I_{x y}(z)=\left(I_{Y Y}-I_{X X}\right) \sin \left(\frac{z}{L} \theta_{t}\right) \cos \left(\frac{z}{L} \theta_{t}\right)
\end{aligned}
$$

where $L$ is the length of the pre-twisted beam and the area moments of inertia with respect to the axes $\mathrm{X}$ and $\mathrm{Y}$ are

$$
\begin{aligned}
& I_{X X}(z)=\frac{b_{0} t_{0}^{3}}{12}\left(1+\alpha \frac{z}{L}\right)\left(1+\beta \frac{z}{L}\right)^{3} \\
& I_{Y Y}(z)=\frac{b_{0}^{3} t_{0}}{12}\left(1+\alpha \frac{z}{L}\right)^{3}\left(1+\beta \frac{z}{L}\right)
\end{aligned}
$$

Hamilton's principle of motion is

$$
\int_{t_{1}}^{t_{2}}(\delta T-\delta U+\delta W) d t=0
$$

where $\delta W, \delta T$ and $\delta U$ are the virtual work, the variation of kinetic energy and the variation of strain energy, respectively. By using Hamilton principle, the equations of motion of this pre-twisted beam can be derived as:

$$
\begin{gathered}
\rho A \frac{\partial^{2} u}{\partial t^{2}}+\frac{\partial^{2} E I_{y y}}{\partial z^{2}} \frac{\partial^{2} u}{\partial z^{2}}+2 \frac{\partial E I_{y y}}{\partial z} \frac{\partial^{3} u}{\partial z^{3}}+E I_{y y} \frac{\partial^{4} u}{\partial z^{4}} \\
+\frac{\partial^{2} E I_{x y}}{\partial z^{2}} \frac{\partial^{2} v}{\partial z^{2}}+2 \frac{\partial E I_{x y}}{\partial z} \frac{\partial^{3} v}{\partial z^{3}}+E I_{x y} \frac{\partial^{4} v}{\partial z^{4}}=0 \\
\rho A \frac{\partial^{2} v}{\partial t^{2}}+\frac{\partial^{2} E I_{x x}}{\partial z^{2}} \frac{\partial^{2} v}{\partial z^{2}}+2 \frac{\partial E I_{x x}}{\partial z} \frac{\partial^{3} v}{\partial z^{3}}+E I_{x x} \frac{\partial^{4} v}{\partial z^{4}} \\
+\frac{\partial^{2} E I_{x y}}{\partial z^{2}} \frac{\partial^{2} u}{\partial z^{2}}+2 \frac{\partial E I_{x y}}{\partial z} \frac{\partial^{3} u}{\partial z^{3}}+E I_{x y} \frac{\partial^{4} u}{\partial z^{4}}=0
\end{gathered}
$$

The corresponding boundary conditions of the clampedfree beam are

$$
\begin{aligned}
& u=0 \quad \text { at } z=0 \\
& \frac{\partial u}{\partial z}=0 \text { at } z=0 \\
& v=0 \text { at } z=0 \\
& \frac{\partial v}{\partial z}=0 \text { at } z=0
\end{aligned}
$$

$$
\begin{gathered}
E I_{y y} \frac{\partial^{2} u}{\partial z^{2}}+E I_{x y} \frac{\partial^{2} v}{\partial z^{2}}=0 \text { at } z=L \\
\frac{\partial}{\partial z}\left(E I_{y y} \frac{\partial^{2} u}{\partial z^{2}}+E I_{x y} \frac{\partial^{2} v}{\partial z^{2}}\right)=0 \text { at } z=L \\
E I_{x x} \frac{\partial^{2} v}{\partial z^{2}}+E I_{x y} \frac{\partial^{2} u}{\partial z^{2}}=0 \text { at } z=L \\
\frac{\partial}{\partial z}\left(E I_{x x} \frac{\partial^{2} v}{\partial z^{2}}+E I_{x y} \frac{\partial^{2} u}{\partial z^{2}}\right)=0 \text { at } z=L
\end{gathered}
$$

The corresponding boundary conditions of the simply supported beam are

$$
\begin{aligned}
u=0 \text { at } z & =0 \\
E I_{y y} \frac{\partial^{2} u}{\partial z^{2}}+E I_{x y} \frac{\partial^{2} v}{\partial z^{2}} & =0 \text { at } z=0 \\
v=0 \text { at } z & =0 \\
E I_{x x} \frac{\partial^{2} v}{\partial z^{2}}+E I_{x y} \frac{\partial^{2} u}{\partial z^{2}} & =0 \text { at } z=0 \\
u=0 \text { at } z & =L \\
E I_{y y} \frac{\partial^{2} u}{\partial z^{2}}+E I_{x y} \frac{\partial^{2} v}{\partial z^{2}} & =0 \text { at } z=L \\
v=0 \text { at } z & =L \\
E I_{x x} \frac{\partial^{2} v}{\partial z^{2}}+E I_{x y} \frac{\partial^{2} u}{\partial z^{2}} & =0 \text { at } z=L
\end{aligned}
$$

The corresponding boundary conditions of the clampedclamped beam are

$$
\begin{gathered}
u=0 \text { at } z=0 \\
\frac{\partial u}{\partial z}=0 \text { at } z=0 \\
v=0 \text { at } z=0 \\
\frac{\partial v}{\partial z}=0 \text { at } z=0 \\
u=0 \text { at } z=L \\
\frac{\partial u}{\partial z}=0 \text { at } z=L \\
v=0 \text { at } z=L \\
\frac{\partial v}{\partial z}=0 \text { at } z=L
\end{gathered}
$$

The system is composed of eight boundary conditions and two coupled governing equations. With the solution assumed to be of the form $u=U \exp (i \omega t)$ and $v=V \exp (i \omega t)$, Eqs. (17) and (18) can then be simplified to 


$$
\begin{aligned}
& \frac{d^{2} E I_{y y}}{d z^{2}} \frac{d^{2} U}{d z^{2}}+2 \frac{d E I_{y y}}{d z} \frac{d^{3} U}{d z^{3}}+E I_{y y} \frac{d^{4} U}{d z^{4}} \\
& +\frac{d^{2} E I_{x y}}{d z^{2}} \frac{d^{2} V}{d z^{2}}+2 \frac{d E I_{x y}}{d z} \frac{d^{3} V}{d z^{3}}+E I_{x y} \frac{d^{4} V}{d z^{4}}=\omega^{2} \rho A U \\
& \frac{d^{2} E I_{x x}}{d z^{2}} \frac{d^{2} V}{d z^{2}}+2 \frac{d E I_{x x}}{d z} \frac{d^{3} V}{d z^{3}}+E I_{x x} \frac{d^{4} V}{d z^{4}} \\
& +\frac{d^{2} E I_{x y}}{d z^{2}} \frac{d^{2} U}{d z^{2}}+2 \frac{d E I_{x y}}{d z} \frac{d^{3} U}{d z^{3}}+E I_{x y} \frac{d^{4} U}{d z^{4}}=\omega^{2} \rho A V
\end{aligned}
$$

where $\omega$ is the angular frequency of vibration. The corresponding boundary conditions of the clamped-free beam are:

$$
\begin{gathered}
U=0 \text { for } z=0 \\
\frac{d U}{d z}=0 \text { for } z=0 \\
V=0 \text { for } z=0 \\
\frac{d V}{d z}=0 \text { for } z=0 \\
E I_{y y} \frac{d^{2} U}{d z^{2}}+E I_{x y} \frac{d^{2} V}{d z^{2}}=0 \text { for } z=L \\
\frac{d}{d z}\left(E I_{y y} \frac{d^{2} U}{d z^{2}}+E I_{x y} \frac{d^{2} V}{d z^{2}}\right)=0 \text { for } z=L \\
E I_{x x} \frac{d^{2} V}{d z^{2}}+E I_{x y} \frac{d^{2} U}{d z^{2}}=0 \text { for } z=L \\
\frac{d}{d z}\left(E I_{x x} \frac{d^{2} V}{d z^{2}}+E I_{x y} \frac{d^{2} U}{d z^{2}}\right)=0 \text { for } z=L
\end{gathered}
$$

The corresponding boundary conditions of the simple supported beam can be shown as

$$
\begin{aligned}
U=0 \text { for } z & =0 \\
E I_{y y} \frac{d^{2} U}{d z^{2}}+E I_{x y} \frac{d^{2} V}{d z^{2}} & =0 \text { for } z=0 \\
V=0 \text { for } z & =0 \\
E I_{x x} \frac{d^{2} V}{d z^{2}}+E I_{x y} \frac{d^{2} U}{d z^{2}} & =0 \text { for } z=0 \\
U=0 \text { for } z & =L \\
E I_{y y} \frac{d^{2} U}{d z^{2}}+E I_{x y} \frac{d^{2} V}{d z^{2}} & =0 \text { for } z=L \\
V=0 \text { for } z & =L \text { for } z=L \\
E I_{x x} \frac{d^{2} V}{d z^{2}}+E I_{x y} \frac{d^{2} U}{d z^{2}} & =0 \text { for }
\end{aligned}
$$

The corresponding boundary conditions of the clampedclamped beam may be presented as stated below:

$$
\begin{aligned}
& U=0 \text { for } z=0 \\
& \frac{d U}{d z}=0 \text { for } z=0 \\
& V=0 \text { for } z=0 \\
& \frac{d V}{d z}=0 \text { for } z=0 \\
& U=0 \text { for } z=L \\
& \frac{d U}{d z}=0 \text { for } z=L \\
& V=0 \text { for } z=L \\
& \frac{d V}{d z}=0 \text { for } z=L
\end{aligned}
$$

In seeking an efficient discretization technique to acquire an accurate numerical solution with very small number of knots, the spline collocation method is utilized to solve numerically these partial differential equations. By applying the spline collocation method, Eqs. (4) and (5) are substituted into (22) and (23). The equation of motion of a pre-twisted beam can be rearranged into the spline collocation method formula. This leads to

$$
\begin{gathered}
{\left[\frac{d^{2} E I_{y y}\left(z_{i}\right)}{d z^{2}} \frac{d^{2} B_{u,-2}\left(z_{i}\right)}{d z^{2}} \frac{d^{2} E I_{y y}\left(z_{i}\right)}{d z^{2}} \frac{d^{2} B_{u,-1}\left(z_{i}\right)}{d z^{2}}\right.} \\
\left.\frac{d^{2} E I_{y y}\left(z_{i}\right)}{d z^{2}} \frac{d^{2} B_{u, N+2}\left(z_{i}\right)}{d z^{2}}\right]\left[\begin{array}{llll}
a_{u,-2} & a_{u,-1} & \ldots & a_{u, N+2}
\end{array}\right]^{T}+
\end{gathered}
$$$$
\left[2 \frac{d E I_{y y}\left(z_{i}\right)}{d z} \frac{d^{3} B_{u,-2}\left(z_{i}\right)}{d z^{3}} 2 \frac{d E I_{y y}\left(z_{i}\right)}{d z} \frac{d^{3} B_{u,-1}\left(z_{i}\right)}{d z^{3}}\right.
$$$$
\left.2 \frac{d E I_{y y}\left(z_{i}\right)}{d z} \frac{d^{3} B_{u, N+2}\left(z_{i}\right)}{d z^{3}}\right]\left[\begin{array}{llll}
a_{u,-2} & a_{u,-1} & \ldots & a_{u, N+2}
\end{array}\right]^{T}+
$$$$
\left[E I_{y y}\left(z_{i}\right) \frac{d^{4} B_{u,-2}\left(z_{i}\right)}{d z^{4}} \quad E I_{y y}\left(z_{i}\right) \frac{d^{4} B_{u,-1}\left(z_{i}\right)}{d z^{4}}\right.
$$$$
\left.E I_{y y}\left(z_{i}\right) \frac{d^{4} B_{u, N+2}\left(z_{i}\right)}{d z^{4}}\right]\left[\begin{array}{llll}
a_{u,-2} & a_{u,-1} & \ldots & a_{u, N+2}
\end{array}\right]^{T}+
$$$$
\left[\frac{d^{2} E I_{x y}\left(z_{i}\right)}{d z^{2}} \frac{d^{2} B_{v,-2}\left(z_{i}\right)}{d z^{2}} \quad \frac{d^{2} E I_{x y}\left(z_{i}\right)}{d z^{2}} \frac{d^{2} B_{v,-1}\left(z_{i}\right)}{d z^{2}}\right.
$$

$$
\left.\frac{d^{2} E I_{x y}\left(z_{i}\right)}{d z^{2}} \frac{d^{2} B_{v, N+2}\left(z_{i}\right)}{d z^{2}}\right]\left[\begin{array}{llll}
a_{u,-2} & a_{u,-1} & \ldots & a_{u, N+2}
\end{array}\right]^{T}+
$$




$$
\begin{aligned}
& {\left[2 \frac{d E I_{x y}\left(z_{i}\right)}{d z} \frac{d^{3} B_{v,-2}\left(z_{i}\right)}{d z^{3}} 2 \frac{d E I_{x y}\left(z_{i}\right)}{d z} \frac{d^{3} B_{v,-1}\left(z_{i}\right)}{d z^{3}}\right.} \\
& +\left[E I_{x y}\left(z_{i}\right) \frac{d^{4} B_{u,-2}\left(z_{i}\right)}{d z^{4}} \quad E I_{x y}\left(z_{i}\right) \frac{d^{4} B_{u,-1}\left(z_{i}\right)}{d z^{4}}\right. \\
& \left.2 \frac{d E I_{x y}\left(z_{i}\right)}{d z} \frac{d^{3} B_{v, N+2}\left(z_{i}\right)}{d z^{3}}\right]\left[\begin{array}{llll}
a_{u,-2} & a_{u,-1} & \ldots & a_{u, N+2}
\end{array}\right]^{T}+ \\
& {\left[E I_{x y}\left(z_{i}\right) \frac{d^{4} B_{v,-2}\left(z_{i}\right)}{d z^{4}} \quad E I_{x y}\left(z_{i}\right) \frac{d^{4} B_{v,-1}\left(z_{i}\right)}{d z^{4}}\right.} \\
& \left.E I_{x y}\left(z_{i}\right) \frac{d^{4} B_{v, N+2}\left(z_{i}\right)}{d z^{4}}\right]\left[\begin{array}{llll}
a_{u,-2} & a_{u,-1} & \ldots & a_{u, N+2}
\end{array}\right]^{T}= \\
& \left.E I_{x y}\left(z_{i}\right) \frac{d^{4} B_{u, N+2}\left(z_{i}\right)}{d z^{4}}\right]\left[\begin{array}{llll}
a_{u,-2} & a_{u,-1} & \ldots & a_{u, N+2}
\end{array}\right]^{T}= \\
& {\left[\omega^{2} \rho A\left(x_{i}\right) B_{v,-2}\left(x_{i}\right) \quad \omega^{2} \rho A\left(x_{i}\right) B_{v,-1}\left(x_{i}\right)\right.} \\
& \left.\omega^{2} \rho A\left(x_{i}\right) B_{v, N+2}\left(x_{i}\right)\right]\left[\begin{array}{llll}
a_{v,-2} & a_{v,-1} & \ldots & a_{v, N+2}
\end{array}\right]^{T} \text { for } \\
& i=0,1, \ldots, N
\end{aligned}
$$$$
\left[\omega^{2} \rho A\left(z_{i}\right) B_{u,-2}\left(z_{i}\right) \quad \omega^{2} \rho A\left(z_{i}\right) B_{u,-1}\left(z_{i}\right)\right.
$$$$
\left.\omega^{2} \rho A\left(z_{i}\right) B_{u, N+2}\left(z_{i}\right)\right]\left[\begin{array}{llll}
a_{u,-2} & a_{u,-1} & \ldots & a_{u, N+2}
\end{array}\right]^{T} \text { for }
$$$$
i=0,1, \ldots, N
$$$$
\left[\frac{d^{2} E I_{x x}\left(z_{i}\right)}{d z^{2}} \frac{d^{2} B_{v,-2}\left(z_{i}\right)}{d z^{2}} \frac{d^{2} E I_{x x}\left(z_{i}\right)}{d z^{2}} \frac{d^{2} B_{v,-1}\left(z_{i}\right)}{d z^{2}}\right.
$$$$
\left.\frac{d^{2} E I_{x x}\left(z_{i}\right)}{d z^{2}} \frac{d^{2} B_{v, N+2}\left(z_{i}\right)}{d z^{2}}\right]\left[\begin{array}{llll}
a_{v,-2} & a_{v,-1} & \ldots & a_{v, N+2}
\end{array}\right]^{T}+
$$$$
\left[2 \frac{d E I_{x x}\left(z_{i}\right)}{d z} \frac{d^{3} B_{v,-2}\left(z_{i}\right)}{d z^{3}} \quad 2 \frac{d E I_{x x}\left(z_{i}\right)}{d z} \frac{d^{3} B_{v,-1}\left(z_{i}\right)}{d z^{3}}\right.
$$$$
\left.2 \frac{d E I_{x x}\left(z_{i}\right)}{d z} \frac{d^{3} B_{v, N+2}\left(z_{i}\right)}{d z^{3}}\right]\left[\begin{array}{llll}
a_{v,-2} & a_{v,-1} & \ldots & a_{v, N+2}
\end{array}\right]^{T}+
$$$$
\left[E I_{x x}\left(z_{i}\right) \frac{d^{4} B_{v,-2}\left(z_{i}\right)}{d z^{4}} \quad E I_{x x}\left(z_{i}\right) \frac{d^{4} B_{v,-1}\left(z_{i}\right)}{d z^{4}} \quad \ldots\right.
$$$$
\left.E I_{x x}\left(z_{i}\right) \frac{d^{4} B_{v, N+2}\left(z_{i}\right)}{d z^{4}}\right]\left[\begin{array}{llll}
a_{v,-2} & a_{v,-1} & \ldots & a_{v, N+2}
\end{array}\right]^{T}
$$$$
+\left[\frac{d^{2} E I_{x y}\left(z_{i}\right)}{d z^{2}} \frac{d^{2} B_{u,-2}\left(z_{i}\right)}{d z^{2}} \frac{d^{2} E I_{x y}\left(z_{i}\right)}{d z^{2}} \frac{d^{2} B_{u,-1}\left(z_{i}\right)}{d z^{2}}\right.
$$$$
\left.\frac{d^{2} E I_{x y}\left(z_{i}\right)}{d z^{2}} \frac{d^{2} B_{u, N+2}\left(z_{i}\right)}{d z^{2}}\right]\left[\begin{array}{llll}
a_{u,-2} & a_{u,-1} & \ldots & a_{u, N+2}
\end{array}\right]^{T}+
$$$$
\left[2 \frac{d E I_{x y}\left(z_{i}\right)}{d z} \frac{d^{3} B_{u,-2}\left(z_{i}\right)}{d z^{3}} \quad 2 \frac{d E I_{x y}\left(z_{i}\right)}{d z} \frac{d^{3} B_{u,-1}\left(z_{i}\right)}{d z^{3}}\right.
$$$$
\left.2 \frac{d E I_{x x}\left(z_{i}\right)}{d z} \frac{d^{3} B_{v, N+2}\left(z_{i}\right)}{d z^{3}}\right]\left[\begin{array}{llll}
a_{u,-2} & a_{u,-1} & \ldots & a_{u, N+2}
\end{array}\right]^{T}
$$

Using the spline collocation method, the boundary conditions of the clamped-free beam can be rearranged into the matrix forms as

$$
\begin{aligned}
& {\left[B_{u,-2}\left(z_{0}\right) \quad B_{u,-1}\left(z_{0}\right) \quad \ldots \quad B_{u, N+2}\left(z_{0}\right)\right]} \\
& {\left[\begin{array}{llll}
a_{u,-2} & a_{u,-1} & \ldots & a_{u, N+2}
\end{array}\right]^{T}=[0]} \\
& {\left[\begin{array}{llll}
\frac{d B_{u,-2}\left(z_{0}\right)}{d z} & \frac{d B_{u,-1}\left(z_{0}\right)}{d z} & \ldots & \frac{d B_{u, N+2}\left(z_{0}\right)}{d z}
\end{array}\right]} \\
& {\left[\begin{array}{llll}
a_{u,-2} & a_{u,-1} & \ldots & a_{u, N+2}
\end{array}\right]^{T}=[0]} \\
& {\left[\begin{array}{llll}
B_{v,-2}\left(z_{0}\right) & B_{v,-1}\left(z_{0}\right) \quad \ldots & B_{v, N+2}\left(z_{0}\right)
\end{array}\right]} \\
& {\left[\begin{array}{llll}
a_{v,-2} & a_{v,-1} & \ldots & a_{v, N+2}
\end{array}\right]^{T}=[0]} \\
& {\left[\begin{array}{llll}
\frac{d B_{v,-2}\left(z_{0}\right)}{d z} & \frac{d B_{v,-1}\left(z_{0}\right)}{d z} & \ldots & \frac{d B_{v, N+2}\left(z_{0}\right)}{d z}
\end{array}\right]} \\
& {\left[\begin{array}{llll}
a_{v,-2} & a_{v,-1} & \ldots & a_{v, N+2}
\end{array}\right]^{T}=[0]} \\
& {\left[E I_{y y}\left(z_{N}\right) \frac{d^{2} B_{u,-2}\left(z_{N}\right)}{d z^{2}} \quad E I_{y y}\left(z_{N}\right) \frac{d^{2} B_{u,-1}\left(z_{N}\right)}{d z^{2}}\right.} \\
& \left.E I_{y y}\left(z_{N}\right) \frac{d^{2} B_{u, N+2}\left(z_{N}\right)}{d z^{2}}\right] \\
& {\left[\begin{array}{llll}
a_{u,-2} & a_{u,-1} & \ldots & a_{u, N+2}
\end{array}\right]^{T}+} \\
& {\left[E I_{x y}\left(z_{N}\right) \frac{d^{2} B_{v,-2}\left(z_{N}\right)}{d z^{2}} \quad E I_{x y}\left(z_{N}\right) \frac{d^{2} B_{v,-1}\left(z_{N}\right)}{d z^{2}}\right.} \\
& \left.E I_{x y}\left(z_{N}\right) \frac{d^{2} B_{v, N+2}\left(z_{N}\right)}{d z^{2}}\right]\left[\begin{array}{llll}
a_{v,-2} & a_{v,-1} & \ldots & a_{v, N+2}
\end{array}\right]^{T}=[0]
\end{aligned}
$$




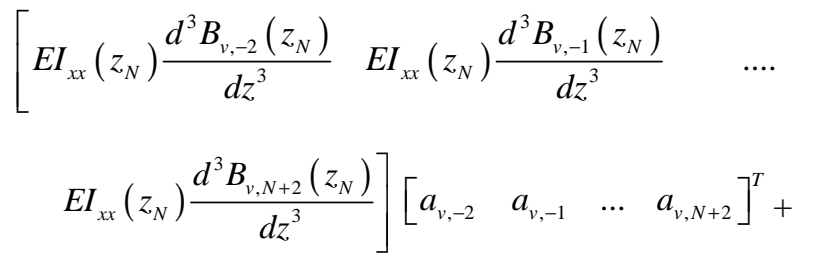

$$
\begin{aligned}
& \begin{array}{c}
{\left[\frac{d E I_{x x}\left(z_{N}\right)}{d z} \frac{d^{2} B_{v,-2}\left(z_{N}\right)}{d z^{2}} \frac{d E I_{x x}\left(z_{N}\right)}{d z} \frac{d^{2} B_{v,-1}\left(z_{N}\right)}{d z^{2}} \quad \ldots\right.} \\
\left.\frac{d E I_{x x}\left(z_{N}\right)}{d z} \frac{d^{2} B_{v, N+2}\left(z_{N}\right)}{d z^{2}}\right]\left[\begin{array}{llll}
a_{v,-2} & a_{v,-1} & \ldots & a_{v, N+2}
\end{array}\right]^{T}+
\end{array} \\
& {\left[E I_{x y}\left(z_{N}\right) \frac{d^{3} B_{u,-2}\left(z_{N}\right)}{d z^{3}}\right.} \\
& E I_{x y}\left(z_{N}\right) \frac{d^{3} B_{u,-1}\left(z_{N}\right)}{d z^{3}} \\
& \left.E I_{x y}\left(z_{N}\right) \frac{d^{3} B_{u, N+2}\left(z_{N}\right)}{d z^{3}}\right]\left[\begin{array}{llll}
a_{u,-2} & a_{u,-1} & \ldots & a_{u, N+2}
\end{array}\right]^{T}+ \\
& {\left[\frac{d E I_{x y}\left(z_{N}\right)}{d z} \frac{d^{2} B_{u,-2}\left(z_{N}\right)}{d z^{2}} \frac{d E I_{x y}\left(z_{N}\right)}{d z} \frac{d^{2} B_{u,-1}\left(z_{N}\right)}{d z^{2}}\right.} \\
& \left.\frac{d E I_{x y}\left(z_{N}\right)}{d z} \frac{d^{2} B_{N+2}\left(z_{N}\right)}{d z^{2}}\right]\left[\begin{array}{llll}
a_{u,-2} & a_{u,-1} & \ldots & a_{u, N+2}
\end{array}\right]^{T}=[0]
\end{aligned}
$$

$$
\begin{aligned}
& {\left[\begin{array}{lll}
E I_{x x}\left(z_{N}\right) \frac{d^{2} B_{v,-2}\left(z_{N}\right)}{d z^{2}} & E I_{x x}\left(z_{N}\right) \frac{d^{2} B_{v,-1}\left(z_{N}\right)}{d z^{2}} \quad \ldots \\
E I_{x x}\left(z_{N}\right) \frac{d^{2} B_{v, N+2}\left(z_{N}\right)}{d z^{2}}
\end{array}\right]} \\
& {\left[\begin{array}{llll}
a_{v,-2} & a_{v,-1} & \ldots & a_{v, N+2}
\end{array}\right]^{T}+} \\
& {\left[\begin{array}{lll}
E I_{x y}\left(z_{N}\right) \frac{d^{2} B_{u,-2}\left(z_{N}\right)}{d z^{2}} & E I_{x y}\left(z_{N}\right) \frac{d^{2} B_{u,-1}\left(z_{N}\right)}{d z^{2}} & \ldots \\
E I_{x y}\left(z_{N}\right) \frac{d^{2} B_{u, N+2}\left(z_{N}\right)}{d z^{2}}
\end{array}\right]\left[\begin{array}{llll}
a_{u,-2} & a_{u,-1} & \ldots & a_{u, N+2}
\end{array}\right]^{T}=[0]}
\end{aligned}
$$

$$
\begin{gathered}
{\left[E I_{x x}\left(z_{N}\right) \frac{d^{3} B_{v,-2}\left(z_{N}\right)}{d z^{3}} \quad E I_{x x}\left(z_{N}\right) \frac{d^{3} B_{v,-1}\left(z_{N}\right)}{d z^{3}} \quad \ldots\right.} \\
\left.E I_{x x}\left(z_{N}\right) \frac{d^{3} B_{v, N+2}\left(z_{N}\right)}{d z^{3}}\right]\left[\begin{array}{llll}
a_{v,-2} & a_{v,-1} & \ldots & a_{v, N+2}
\end{array}\right]^{T}+
\end{gathered}
$$

$$
\begin{aligned}
& {\left[\frac{d E I_{x x}\left(z_{N}\right)}{d z} \frac{d^{2} B_{v,-2}\left(z_{N}\right)}{d z^{2}} \frac{d E I_{x x}\left(z_{N}\right)}{d z} \frac{d^{2} B_{v,-1}\left(z_{N}\right)}{d z^{2}}\right.} \\
& \left.\frac{d E I_{x x}\left(z_{N}\right)}{d z} \frac{d^{2} B_{v, N+2}\left(z_{N}\right)}{d z^{2}}\right]\left[\begin{array}{llll}
a_{v,-2} & a_{v,-1} & \ldots & a_{v, N+2}
\end{array}\right]^{T}+ \\
& {\left[E I_{x y}\left(z_{N}\right) \frac{d^{3} B_{u,-2}\left(z_{N}\right)}{d z^{3}} \quad E I_{x y}\left(z_{N}\right) \frac{d^{3} B_{u,-1}\left(z_{N}\right)}{d z^{3}}\right.} \\
& \left.E I_{x y}\left(z_{N}\right) \frac{d^{3} B_{u, N+2}\left(z_{N}\right)}{d z^{3}}\right]\left[\begin{array}{llll}
a_{u,-2} & a_{u,-1} & \ldots & a_{u, N+2}
\end{array}\right]^{T}+ \\
& {\left[\frac{d E I_{x y}\left(z_{N}\right)}{d z} \frac{d^{2} B_{u,-2}\left(z_{N}\right)}{d z^{2}} \frac{d E I_{x y}\left(z_{N}\right)}{d z} \frac{d^{2} B_{u,-1}\left(z_{N}\right)}{d z^{2}}\right.} \\
& \left.\frac{d E I_{x y}\left(z_{N}\right)}{d z} \frac{d^{2} B_{u, N+2}\left(z_{N}\right)}{d z^{2}}\right]\left[\begin{array}{llll}
a_{u,-2} & a_{u,-1} & \ldots & a_{u, N+2}
\end{array}\right]^{T}=[0]
\end{aligned}
$$

Using the spline collocation method, the boundary conditions of the simple supported beam can be rearranged into the matrix forms as

$$
\begin{aligned}
& {\left[B_{u,-2}\left(z_{0}\right) \quad B_{u,-1}\left(z_{0}\right) \quad \ldots \quad B_{u, N+2}\left(z_{0}\right)\right]} \\
& {\left[\begin{array}{llll}
a_{u,-2} & a_{u,-1} & \ldots & a_{u, N+2}
\end{array}\right]^{T}=[0]} \\
& {\left[E I_{y y}\left(z_{0}\right) \frac{d^{2} B_{u,-2}\left(z_{0}\right)}{d z^{2}} \quad E I_{y y}\left(z_{0}\right) \frac{d^{2} B_{u,-1}\left(z_{0}\right)}{d z^{2}}\right.} \\
& \left.\ldots \quad E I_{y y}\left(z_{0}\right) \frac{d^{2} B_{u, N+2}\left(z_{0}\right)}{d z^{2}}\right]\left[\begin{array}{llll}
a_{u,-2} & a_{u,-1} & \ldots & a_{u, N+2}
\end{array}\right]^{T}+ \\
& {\left[E I_{x y}\left(z_{0}\right) \frac{d^{2} B_{v,-2}\left(z_{0}\right)}{d z^{2}} \quad E I_{x y}\left(z_{0}\right) \frac{d^{2} B_{v,-1}\left(z_{0}\right)}{d z^{2}}\right.} \\
& \left.\ldots \quad E I_{x y}\left(z_{0}\right) \frac{d^{2} B_{v, N+2}\left(z_{0}\right)}{d z^{2}}\right]\left[\begin{array}{llll}
a_{v,-2} & a_{v,-1} & \ldots & a_{v, N+2}
\end{array}\right]^{T}=[0]
\end{aligned}
$$

$$
\begin{aligned}
& {\left[\begin{array}{llll}
B_{v,-2}\left(z_{0}\right) & B_{v,-1}\left(z_{0}\right) & \ldots & B_{v, N+2}\left(z_{0}\right)
\end{array}\right]} \\
& {\left[\begin{array}{llll}
a_{v,-2} & a_{v,-1} & \ldots & a_{v, N+2}
\end{array}\right]^{T}=[0]} \\
& {\left[\begin{array}{lll}
E I_{x x}\left(z_{0}\right) \frac{d^{2} B_{v,-2}\left(z_{0}\right)}{d z^{2}} & E I_{x x}\left(z_{0}\right) \frac{d^{2} B_{v,-1}\left(z_{0}\right)}{d z^{2}}
\end{array}\right.}
\end{aligned}
$$




$$
\begin{aligned}
& \left.\ldots \quad E I_{x x}\left(z_{0}\right) \frac{d^{2} B_{v, N+2}\left(z_{0}\right)}{d z^{2}}\right]\left[\begin{array}{llll}
a_{v,-2} & a_{v,-1} & \ldots & a_{v, N+2}
\end{array}\right]^{T}+ \\
& {\left[E I_{x y}\left(z_{0}\right) \frac{d^{2} B_{u,-2}\left(z_{0}\right)}{d z^{2}} \quad E I_{x y}\left(z_{0}\right) \frac{d^{2} B_{u,-1}\left(z_{0}\right)}{d z^{2}}\right.} \\
& \left.\ldots \quad E I_{x y}\left(z_{0}\right) \frac{d^{2} B_{u, N+2}\left(z_{0}\right)}{d z^{2}}\right]\left[\begin{array}{llll}
a_{u,-2} & a_{u,-1} & \ldots & a_{u, N+2}
\end{array}\right]^{T}=[0]
\end{aligned}
$$

$$
\left[\begin{array}{llll}
B_{u,-2}\left(z_{N}\right) & B_{u,-1}\left(z_{N}\right) \quad \ldots \quad B_{u, N+2}\left(z_{N}\right)
\end{array}\right]
$$$$
\left[\begin{array}{llll}
a_{u,-2} & a_{u,-1} & \ldots & a_{u, N+2}
\end{array}\right]^{T}=[0]
$$

$$
\begin{aligned}
& {\left[E I_{y y}\left(z_{N}\right) \frac{d^{2} B_{u,-2}\left(z_{N}\right)}{d z^{2}} \quad E I_{y y}\left(z_{N}\right) \frac{d^{2} B_{u,-1}\left(z_{N}\right)}{d z^{2}}\right.} \\
& \left.\ldots \quad E I_{y y}\left(z_{N}\right) \frac{d^{2} B_{u, N+2}\left(z_{N}\right)}{d z^{2}}\right]\left[\begin{array}{llll}
a_{u,-2} & a_{u,-1} & \ldots & a_{u, N+2}
\end{array}\right]^{T}+ \\
& {\left[E I_{x y}\left(z_{N}\right) \frac{d^{2} B_{v,-2}\left(z_{N}\right)}{d z^{2}} \quad E I_{x y}\left(z_{N}\right) \frac{d^{2} B_{v,-1}\left(z_{N}\right)}{d z^{2}}\right.} \\
& \left.\ldots \quad E I_{x y}\left(z_{N}\right) \frac{d^{2} B_{v, N+2}\left(z_{N}\right)}{d z^{2}}\right]\left[\begin{array}{llll}
a_{v,-2} & a_{v,-1} & \ldots & a_{v, N+2}
\end{array}\right]^{T}=[0]
\end{aligned}
$$

$$
\left[B_{v,-2}\left(z_{N}\right) \quad B_{v,-1}\left(z_{N}\right) \quad \ldots \quad B_{v, N+2}\left(z_{N}\right)\right]
$$$$
\left[\begin{array}{llll}
a_{v,-2} & a_{v,-1} & \ldots & a_{v, N+2}
\end{array}\right]^{T}=[0]
$$

$\left[E I_{x x}\left(z_{N}\right) \frac{d^{2} B_{v,-2}\left(z_{N}\right)}{d z^{2}} \quad E I_{x x}\left(z_{N}\right) \frac{d^{2} B_{v,-1}\left(z_{N}\right)}{d z^{2}}\right.$

$\left.\ldots \quad E I_{x x}\left(z_{N}\right) \frac{d^{2} B_{v, N+2}\left(z_{N}\right)}{d z^{2}}\right]\left[\begin{array}{llll}a_{v,-2} & a_{v,-1} & \ldots & a_{v, N+2}\end{array}\right]^{T}+$

$$
\left[E I_{x y}\left(z_{N}\right) \frac{d^{2} B_{u,-2}\left(z_{N}\right)}{d z^{2}} \quad E I_{x y}\left(z_{N}\right) \frac{d^{2} B_{u,-1}\left(z_{N}\right)}{d z^{2}}\right.
$$$$
\left.\ldots \quad E I_{x y}\left(z_{N}\right) \frac{d^{2} B_{u, N+2}\left(z_{N}\right)}{d z^{2}}\right]\left[\begin{array}{llll}
a_{u,-2} & a_{u,-1} & \ldots & a_{u, N+2}
\end{array}\right]^{T}=[0]
$$

Using the spline collocation method, the boundary conditions of the clamped-clamped beam can be rearranged into the matrix forms as

$$
\begin{aligned}
& {\left[\begin{array}{llll}
B_{u,-2}\left(z_{0}\right) & B_{u,-1}\left(z_{0}\right) \quad \ldots & B_{u, N+2}\left(z_{0}\right)
\end{array}\right]} \\
& {\left[\begin{array}{llll}
a_{u,-2} & a_{u,-1} & \ldots & a_{u, N+2}
\end{array}\right]^{T}=[0]} \\
& {\left[\begin{array}{llll}
\frac{d B_{u,-2}\left(z_{0}\right)}{d z} & \frac{d B_{u,-1}\left(z_{0}\right)}{d z} & \ldots & \frac{d B_{u, N+2}\left(z_{0}\right)}{d z}
\end{array}\right]} \\
& {\left[\begin{array}{llll}
a_{u,-2} & a_{u,-1} & \ldots & a_{u, N+2}
\end{array}\right]^{T}=[0]} \\
& {\left[\begin{array}{llll}
B_{v,-2}\left(z_{0}\right) & B_{v,-1}\left(z_{0}\right) \quad \ldots & B_{v, N+2}\left(z_{0}\right)
\end{array}\right]} \\
& {\left[\begin{array}{llll}
a_{v,-2} & a_{v,-1} & \ldots & a_{v, N+2}
\end{array}\right]^{T}=[0]} \\
& {\left[\begin{array}{llll}
\frac{d B_{v,-2}\left(z_{0}\right)}{d z} & \frac{d B_{v,-1}\left(z_{0}\right)}{d z} & \ldots & \frac{d B_{v, N+2}\left(z_{0}\right)}{d z}
\end{array}\right]} \\
& {\left[\begin{array}{llll}
a_{v,-2} & a_{v,-1} & \ldots & a_{v, N+2}
\end{array}\right]^{T}=[0]} \\
& {\left[\begin{array}{llll}
B_{u,-2}\left(z_{N}\right) & \left.B_{u,-1}\left(z_{N}\right) \quad \ldots \quad B_{u, N+2}\left(z_{N}\right)\right]
\end{array}\right.} \\
& {\left[\begin{array}{llll}
a_{u,-2} & a_{u,-1} & \ldots & a_{u, N+2}
\end{array}\right]^{T}=[0]} \\
& {\left[\begin{array}{llll}
\frac{d B_{u,-2}\left(z_{N}\right)}{d z} & \frac{d B_{u,-1}\left(z_{N}\right)}{d z} & \ldots & \frac{d B_{u, N+2}\left(z_{N}\right)}{d z}
\end{array}\right]}
\end{aligned}
$$$$
\left[\begin{array}{llll}
a_{u,-2} & a_{u,-1} & \ldots & a_{u, N+2}
\end{array}\right]^{T}=[0]
$$

$$
\left[\begin{array}{llll}
B_{v,-2}\left(z_{N}\right) & B_{v,-1}\left(z_{N}\right) \quad \ldots \quad B_{v, N+2}\left(z_{N}\right)
\end{array}\right]
$$$$
\left[\begin{array}{llll}
a_{v,-2} & a_{v,-1} & \ldots & a_{v, N+2}
\end{array}\right]^{T}=[0]
$$

$$
\begin{aligned}
& {\left[\begin{array}{llll}
\frac{d B_{v,-2}\left(z_{N}\right)}{d z} & \frac{d B_{v,-1}\left(z_{N}\right)}{d z} & \ldots & \frac{d B_{v, N+2}\left(z_{N}\right)}{d z}
\end{array}\right]} \\
& {\left[\begin{array}{llll}
a_{v,-2} & a_{v,-1} & \ldots & a_{v, N+2}
\end{array}\right]^{T}=[0]}
\end{aligned}
$$

The eigenvalues of the resultant algebraic equation system provide the natural frequencies of the pre-twisted beam problem.

\section{NUMERICAL RESULTS AND DISCUSSION}

Figure 2 shows the calculated natural frequencies of clamped-free beams with different pre-twisted angles. The data for this pre-twisted beam are [4]: $b_{0} / t_{0}=4$. The non-dimensional natural frequencies of the pre-twisted beam are defined as $\bar{\omega}_{i}=\omega_{i} \sqrt{12 \rho A L^{4} / E b_{0} t_{0}^{3}}$. Numerical results 


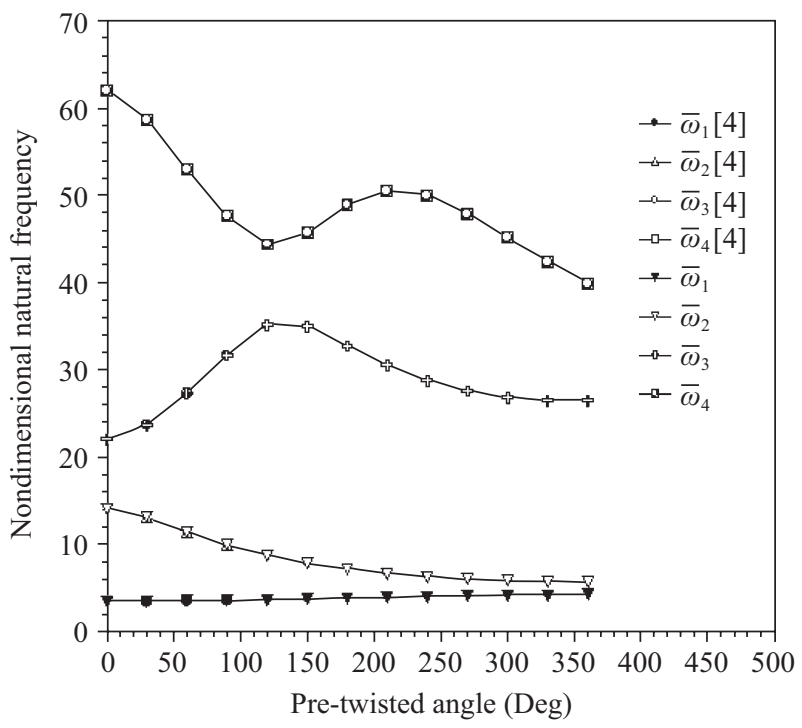

Fig. 2. The calculated natural frequencies of the clamped-free beams with different pre-twisted angles.

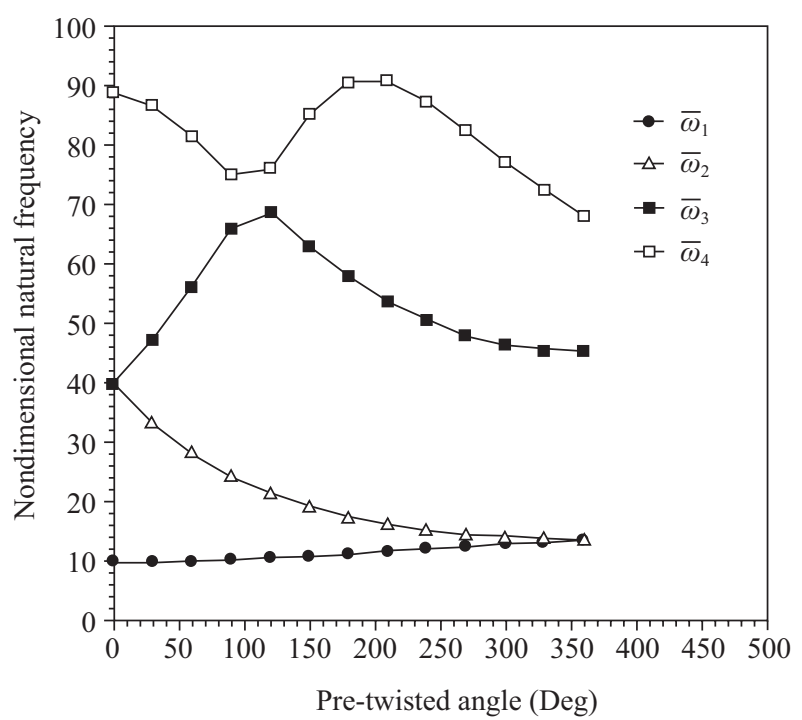

Fig. 3. The natural frequencies of the simple-supported beams with various pre-twisted angles.

indicate that the natural frequencies of a pre-twisted beam calculated using the spline collocation method are shown to be in favorable agreement with the numerical results solved using the Rayleigh-Ritz method [4]. No significant error is found for the results calculated using the spline collocation method. Results indicated that a higher first natural frequency is calculated for the beam with a higher total pre-twisted angle for $\theta_{t}<360^{\circ}$. Numerical results also indicated that the calculated second natural frequency is decreased while the pre-twisted angle increasing for $\theta_{t}<360^{\circ}$.

Figure 3 shows the natural frequencies of simple-supported beams with various pre-twisted angles. Numerical results indicated that a higher first natural frequency is calculated for the beam

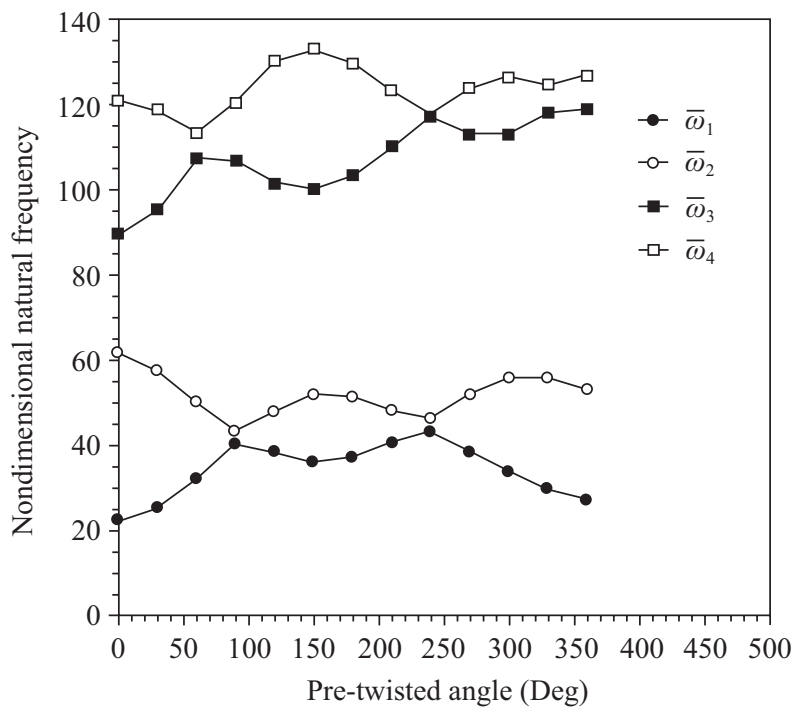

Fig. 4. The natural frequencies of the clamped-clamped beams with various pre-twisted angles.

with a higher pre-twisted angle for $\theta_{t}<360^{\circ}$. Numerical results also indicated that the calculated second natural frequency is decreased while the pre-twisted angle increasing for $\theta_{t}<360^{\circ}$. The pre-twisted angles deeply affect the third and fourth natural frequencies.

Figure 4 shows the natural frequencies of the clampedclamped beams with various pre-twisted angles. Numerical results indicated that a higher first natural frequency is calculated for the beam with a higher pre-twisted angle for $\theta_{t}<90^{\circ}$. Numerical results also indicated that the calculated second natural frequency is decreased while the pre-twisted angle increasing for $\theta_{t}<90^{\circ}$. Numerical results in this example show that the pre-twisted angle can significantly affect the natural frequencies of the pre-twisted beams.

Figure 5 describes the natural frequencies of the clampedfree beams with various tapered angles $\beta$. The fundamental frequencies are almost constant. Numerical results also indicated that the calculated second, third and fourth natural frequencies are increased while the tapered angles increasing.

Figure 6 shows the natural frequencies of the simplesupported beams with various tapered angles $\beta$. Numerical results in this example show that the tapered angle can significantly affect the first frequency. The first, second, third and fourth natural frequencies increase with tapered angles $\beta$, almost linearly.

Figure 7 shows the natural frequencies of the clampedclamped beams with various tapered angles $\beta$. Numerical results also indicated that the calculated natural frequencies are increased while the tapered angles increasing in general. Numerical results indicate that the tapered angle is a very sensitive parameter for the vibration of the tapered beam. The clamped-clamped boundary conditions give rise to higher natural frequencies of the beams in comparison with the simply supported boundary conditions. 


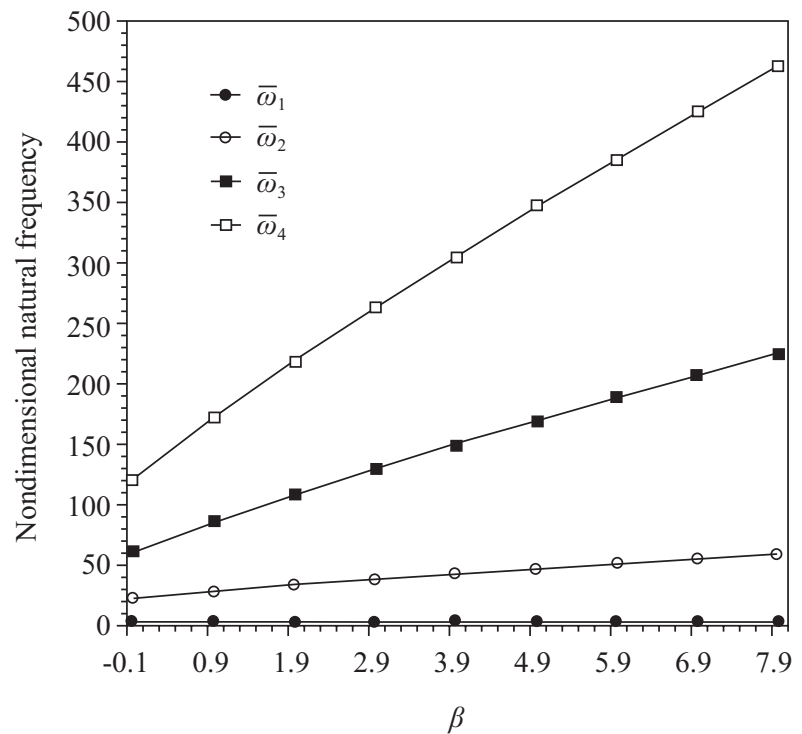

Fig. 5. The natural frequencies of the clamped-free beams with various tapered angles $\beta$.

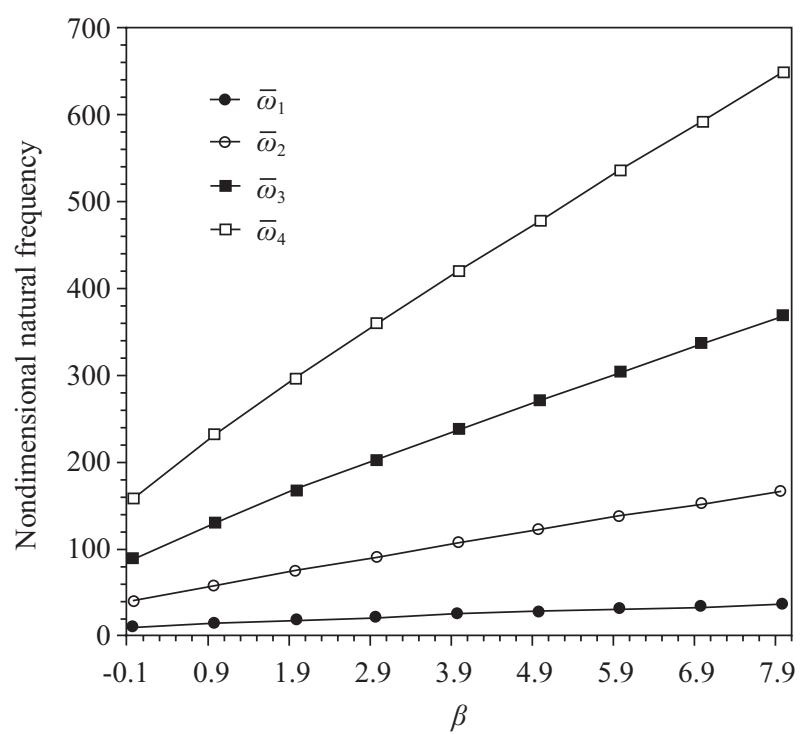

Fig. 6. The natural frequencies of the simple-supported beams for various tapered angles $\beta$.

\section{CONCLUDING REMARKS}

The variation in calculated natural frequencies for the pretwisted beams using the spline collocation method is investigated. The solution of the governing fourth-order differential equation is approximated by the spline function with polynomial. The efficiency and accuracy of the proposed method is ascertained by comparison with existing solutions. $\mathrm{Nu}$ merical results in different cases validated the applicability of the proposed method for solving such an engineering problem. The pre-twisted angles influence the natural frequencies of

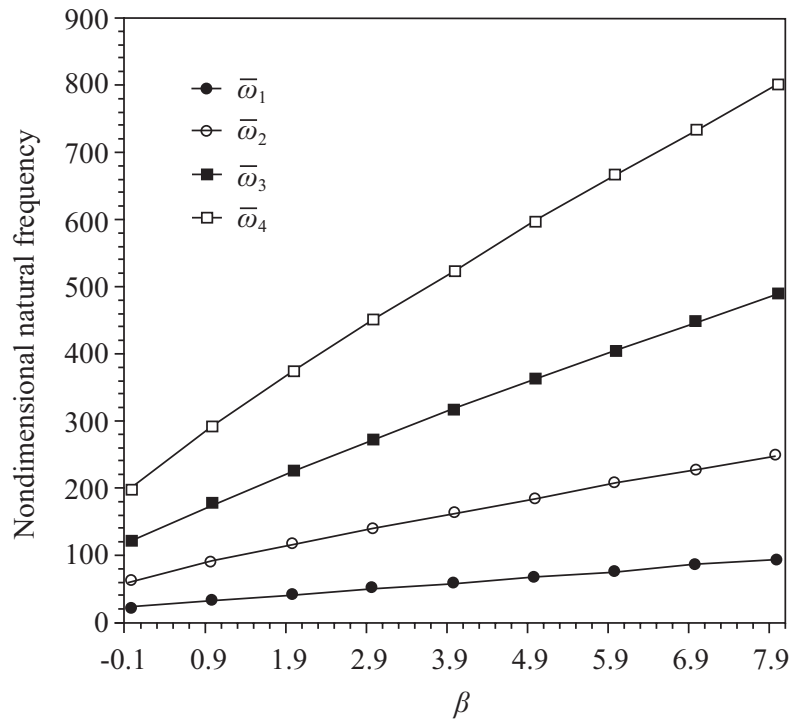

Fig. 7. The natural frequencies of the clamped-clamped beams with various tapered angles $\beta$.

the beams. The demonstrated accuracy and simplicity of the proposed method makes it a good candidate for modeling more complicated pre-twisted beam problems.

\section{REFERENCES}

1. Abrate, S., "Vibrations of non-uniform rods and beams," Journal of Sound and Vibration, Vol. 185, pp. 703-716 (1995).

2. Anderson, G. L., "On the extensional and flexural vibration of rotating bars," International Journal of Nonlinear Mechanics, Vol. 10, pp. 223-236 (1975).

3. Bert, C. W. and Sheu, Y., "Static analyses of beams and plates by spline collocation method," Journal of Engineering Mechanics, Vol. 122, pp. 375-378 (1996).

4. Dawson, B., "Couple bending-bending vibrations of pre-twisted cantilever blading treated by Rayleigh-Ritz energy method," Journal of Mechanical Engineering Science, Vol. 10, pp. 381-388 (1968).

5. Dawson, B. and Carneige, W., "Model curves of pretwisted beams of rectangular cross-section," Journal of Mechanical Engineering Science, Vol. 11, pp. 1-13 (1969).

6. El-Hawary, H. M., Zanaty, E. A., and El-Sanousy, E., "Quartic spline collocation methods for elliptic partial differential equations," Applied Mathematics and Computation, Vol. 168, pp. 198-221 (2005).

7. Greville, T. N. E., Theory and Applications of Spline Functions, Academic Press, New York (1969).

8. Gupa, R. S. and Rao, S. S., "Finite element eigenvalue analysis of tapered and twisted Timoshenko beams," Journal of Sound and Vibration, Vol. 56, pp. 187-200 (1978).

9. Hodges, D. H., Chung, Y. Y., and Shang, X. Y., "Discrete transfer matrix method for non-uniform rotating beams," Journal of Sound and Vibration, Vol. 169, pp. 276-283 (1994).

10. Kuang, J. H. and Hsu, M. H., "Eigen solutions of grouped turbo blades solved by the generalized differential quadrature method," Transactions of ASME, Journal of Engineering for Gas Turbines and Power, Vol. 124, pp. 1011-1017 (2002).

11. Kuang, J. H. and Hsu, M. H., "Fiber effect of an orthotropic composite blade solved by the differential quadrature method," Composite Structures, Vol. 58, pp. 457-468 (2002). 
12. Lin, S. M., Wang, W. R., and Lee, S. Y., "The dynamic analysis of nonuniformly pretwisted Timoshenko beams with elastic boundary conditions," International Journal of Mechanical Sciences, Vol. 43, pp. 2385-2406 (2001).

13. Patlashenko, I. and Weller, T., "Two-dimensional spline collocation method for nonlinear analysis of laminated panels," Computers \& Structures, Vol. 57, pp. 131-139 (1995).

14. Prenter, P. M., Spline and Variational Methods, John Wiley \& Sons, Inc., New York (1975).

15. Rao, J. S., "Flexural vibration of pretwisted tapered cantilever blades," ASME Journal of Engineering for Industry, Vol. 94, pp. 343-346 (1972).

16. Rao, J. S., "Vibration of rotating, pretwisted and tapered blades," Mechanism and Machine Theory, Vol. 12, pp. 331-337 (1977).

17. Rao, S. C. S. and Kumar, M., "Optimal B-spline collocation method for self-adjoint singularly perturbed boundary value problems," Applied Mathematics and Computation, Vol. 188, pp. 749-761 (2007).

18. Schumalcer, L., Spline Functions: Basic Theory, Wiley-Interscience, New York (1980).

19. Storiti, D. and Aboelnaga, Y., "Bending vibrations of a class of rotating beams with hypergeometric solutions," Journal of Applied Mechanics, Vol. 54, pp. 311-314 (1987).

20. Subrahmanyam, K. B., Kulkarni, S. V., and Rao, J. S., "Coupled bending-bending vibrations of pre-twisted cantilever blading allowing for shear deflection and rotary inertia by the Reissner method," International Journal of Mechanical Science, Vol. 23, pp. 517-530 (1981).

21. Subrahmanyam, K. B. and Rao, J. S., "Coupled bending-bending vibrations of pre-twisted tapered cantilever beams treated by the Reissner method," Journal of Sound and Vibration, Vol. 82, pp. 577-592 (1982).

22. Swaminathan, M. and Rao, J. S., "Vibrations of rotating, pretwisted and tapered blades," Mechanism and Machine Theory, Vol. 12, pp. 331-337 (1977).

23. Viswanathan, K. K. and Navaneethakrishnan, P. V., "Free vibration study of layered cylindrical shells by collocation with splines," Journal of Sound and Vibration, Vol. 260, pp. 807-827 (2003).

24. Wu, L. Y. and Chen, Y. T., "Analysis of rigid frame by spline collocation method," Journal of the Chinese Institute of Engineers, Vol. 26, pp. 619-634 (2003)

25. Wu, L. Y. and Chen, Y. T., "Application of spline collocation method in analysis of beam and continuous beam," The Chinese Journal of Mechanics-Series A, Vol. 19, pp. 319-326 (2003).

26. Wu, L. Y., Chung, L. L., Chen, Y. T., Wu, T. Y., and Wu, T. J., "Application of modified spline collocation method in analysis of rigid frame," Proceedings of the Ninth International Conference on Computing in Civil and Building Engineering, Vol. 1, pp. 327-332 (2002).

27. Young, T. H., "Dynamic response of a pre-twisted tapered beam with non-constant rotating speed," Journal of Sound and Vibration, Vol. 150, pp. 435-446 (1991). 